\title{
INFRARED ENVIRONMENT OF THE BE STAR 6 CEPHEI: INTERACTION OF STELLAR AND INTERSTELLAR WINDS
}

\author{
M. Kun \\ Konkoly Observatory, Hungary
}

\begin{abstract}
Several arclike structures are seen on the high resolution IRAS images (processes via GEISHA system of Groningen) centred on the Be star 6 Cephei, among others an extended bow shock which indicates the rapd motion of the star and the interstellar matter relative to each other. 6 Cephei is embedded in a giant infrared-emitting shell which is thougth to be a three million year old supernova remnant, therefore in this case the presence of the bow shock around the star shows the interaction of a supernova blast wave with the stellar wind of a $\mathrm{Be}$ star. In this paper an attempt is made to reveal the history of the interactions of the star with its surroundings during different periods of its life which are recorded on the infrared maps. Some aspects of the formation and evolution of 6 Cephei can be inferred also from its infrared environment.
\end{abstract}

